\title{
Contribution to the bryoflora of the Ciężkowickie Foothills (Western Carpathians, Poland)
}

Adam Stebel \& Grzegorz Vončina

Contribution to the bryoflora of the Ciężkowickie Foothills (Western Carpathians, Poland). - Acta Mus. Siles. Sci. Natur., 66: 121-135, 2017.

\begin{abstract}
The paper provides information about new localities of 118 species, including 2 hornworts, 22 liverworts and 94 mosses collected at the Ciężkowickie Foothills. The most interesting are bryophytes protected and threatened in Poland, for example Fossombronia wondraczekii, Nowellia curvifolia, Sphagnum compactum, Trichocolea tomentella and Weissia squarrosa.
\end{abstract}

Key words: hornworts, liverworts, mosses, distributional data, threatened species, protected species, Ciężkowickie Foothills, Western Carpathians, Poland

\section{Introduction}

Ciężkowickie Foothills (Pogórze Ciężkowickie in Polish) is a region lies in the Western Carpathians (Fig. 1) and covers an area of $740 \mathrm{~km}^{2}$ (Kondracki 1994). It is densely populated and meadows, pastures, arable fields and villages, with rather small patches of forests predominate in its landscape (Fig. 2, 3). The highest point is Mt. Liwocz (562 m a.s.1.). Ciężkowickie Foothills region is one of the poorest bryologically known area in the Polish part of the Carpathians and only several sites of bryophytes are known from here (Szafran 1956, Armata 2006, Cykowska 2006a,b).

The paper provides information about new localities of 118 species, including 2 hornworts, 22 liverworts and 94 mosses.

\section{Materials and methods}

Field investigations were carried out in the years 2011, 2014 and 2016. List of taxa are set alphabetically. The moss species names follow mainly Ochyra et al. (2003), whereas hornworts and liverworts according to Klama (2006a). Species protected by law in Poland were taken after 'Regulation of the Minister of Environment' (2014) whereas threatened after Klama (2006b) and Żarnowiec et al. (2004).

For each species the following information is given: ATMOS grid square (Ochyra \& Szmajda 1981), GPS coordinates, locality, habitat and altitude.

\section{List of species and localities}

Abbreviations: ! - species partly protected in Poland, in the square brackets - species threatened in Poland with threat categories, c. spor. - with sporophytes, c. gem. - with gemmae.

\section{HORNWORTS / ANTHOCEROTOPHYTA}

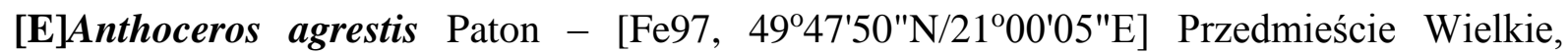
stubble-field, c. spor., 343 m; [Fe98, 4949'35"N/2109'49"E] Żurowa-Wielka Wieś, stubble-field, c. spor., 305 m (Fig. 4).

Phaeoceros carolinianus (Michx.) Prosk. - [Fe98, 4949'35"N/2109'49"E] Żurowa-Wielka Wieś, stubble-field, c. spor., $305 \mathrm{~m}$. 


\section{LIVERWORTS / MARCHANTIOPHYTA}

Aneura pinguis (L.) Dumort. - [Ge08, 49 43'09"N/21 05'24"E] Moszczenica, 'Księży Las' forest, wet mineral soil in Abies alba forest, $340 \mathrm{~m}$.

!Bazzania trilobata (L.) Gray - [Fe97, 4946'39"N/2058'11"E] 'Skamieniałe Miasto' nature reserve, humus soil among Vaccinium myrtillus near sandstone outcrops, $302 \mathrm{~m}$; [Fe97, 49 46'41"N/20 58'47"E] Ciężkowice, 'Wodospad' nature monument, on ground in Abies alba forest, $311 \mathrm{~m}$; [Ge08, 49 $\left.43^{\circ} 05^{\prime \prime} \mathrm{N} / 21^{\circ} 05^{\prime} 35^{\prime \prime} \mathrm{E}\right]$ Moszczenica, 'Księży Las' forest, forest floor in Abies alba forest, $344 \mathrm{~m}$.

Blasia pusilla L. - [Fe87, 49 51'29"N/2103'02"E] Lubaszowa, near road to the monastery, landslide, $255 \mathrm{~m}$.

Calypogeia azurea Stotler \& Crotz - [Fe88, 4950'06"N/2105'31"E] Jodłówka Tuchowska, between Ratówki and Kobyli Dział settlements, wet slope in Abies alba forest, c. spor., 368 m; [Ge08, 49 43'04"N/21 05'20"E] Moszczenica, 'Księży Las' forest, forest floor in Abies alba forest, $349 \mathrm{~m}$.

Cephalozia bicuspidata (L.) Dumort. - [Fe88, 49 50'06"N/2105'31"E] Jodłówka Tuchowska, between Ratówki and Kobyli Dział settlements, log in Abies alba forest, c. spor., 368 m; [Ge08, 49 43'09"N/2105'24"E] Moszczenica, 'Księży Las' forest, rotten wood of silver fir in Abies alba forest, $340 \mathrm{~m}$.

Chiloscyphus polyanthos (L.) Corda - [Fe87, 49 51'26"N/21 $\left.03^{\circ} 01^{\prime \prime E}\right]$ Lubaszowa, near road to the monastery, bank of stream, $255 \mathrm{~m}$; [Ge08, 49043'09"N/21 05'24"E] Moszczenica, 'Księży Las' forest, wet mineral soil, seepage area in Abies alba forest, $340 \mathrm{~m}$.

Conocephalum conicum (L.) Dumort. - [Fe87, 49 51'26"N/21 03'01"E] Lubaszowa, near road to the monastery, bank of stream, $255 \mathrm{~m}$; [Ge08, 49 $\left.43^{\prime} 04^{\prime \prime} \mathrm{N} / 21^{\circ} 05^{\prime} 20^{\prime \prime E}\right]$ Moszczenica, 'Księży Las' forest, mineral soil, stream bed in Abies alba forest, $349 \mathrm{~m}$.

![E] Fossombronia wondraczekii (Corda) Lindb. - [Fe88, 49 $50^{\circ} 42^{\prime \prime N} / 21^{\circ} 08^{\prime} 50$ "E] Żurowa-

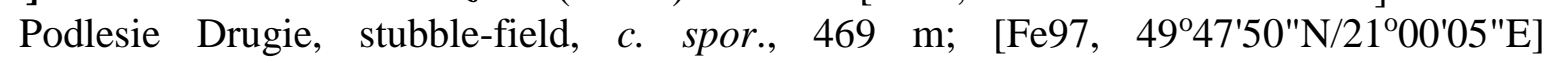
Przedmieście Wielkie, stubble-field, c. spor., $343 \mathrm{~m}$; [Fe98, 4949'35"N/2109'49"E] Żurowa-Wielka Wieś, stubble-field, c. spor., $305 \mathrm{~m}$.

!Frullania dilatata (L.) Dumort. - [Ff90, 49049'14"N/21'23'25"E] Brzyska, bark of Acer platanoides in old park near the castle, $241 \mathrm{~m}$.

Jamesoniella autumnalis (DC.) Steph. - [Ge08, 4943'01"N/2105'26"E] Moszczenica, 'Księży Las' forest, bottom of trunk of silver fir in Abies alba forest, $346 \mathrm{~m}$.

Lepidozia reptans (L.) Dumort. - [Ge08, 4943'04"N/2105'20"E] Moszczenica, 'Księży Las' forest, forest floor in Abies alba forest, $349 \mathrm{~m}$.

Lophocolea bidentata (L.) Dumort. - [Ge08, 49 43'09"N/21 $\left.05^{\prime} 24^{\prime \prime E}\right]$ Moszczenica, 'Księży Las' forest, forest floor in Abies alba forest, $340 \mathrm{~m}$.

Lophocolea heterophylla (Schrad.) Dumort. - [Fe89, 49 51'16"N/21 $\left.{ }^{\circ} 16^{\prime} 28^{\prime \prime E}\right]$ Wisowa, $384 \mathrm{~m}$; [Fe88, 49 $\left.50^{\prime} 05^{\prime \prime N} / 21^{\circ} 05^{\prime} 33^{\prime \prime E}\right]$ Jodłówka Tuchowska, between Ratówki and Kobyli Dział settlements, log in Abies alba forest, 383 m; [Ge08, 49 43'09"N/21 05'24"E] Moszczenica, 'Księży Las' forest, stump in Abies alba forest, $340 \mathrm{~m}$.

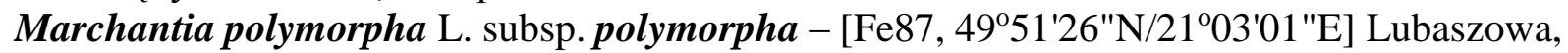
bank of stream, $255 \mathrm{~m}$.

Marchantia polymorpha L. subsp. ruderalis Bischl. \& Boisselier - [Fe88, 49 $52^{\prime} 53^{\prime \prime N} / 21^{\circ} 05^{\prime} 46^{\prime \prime E}$ ] Bistuszowa, wayside, $231 \mathrm{~m}$; [Fe88, 4952'55"N/2112'16"E] Kowalowa, waste place, $281 \mathrm{~m}$.

![V]Nowellia curvifolia (Dicks.) Mitt. - [Ge08, 49²43'03"N/21ㅇ5'13"E] Moszczenica, 'Księży Las' forest, rotten wood in Abies alba forest, $365 \mathrm{~m}$. 
Pellia epiphylla (L.) Corda - [Fe87, 49 $\left.51^{\prime} 26^{\prime \prime} \mathrm{N} / 21^{\circ} 03^{\prime} 01^{\prime \prime E}\right]$ Lubaszowa, near road to the monastery, bank of stream, 255 m; [Ge08, 4943'09"N/21 05'24"E] Moszczenica, 'Księży Las' forest, moist mineral soil in Abies alba forest, $340 \mathrm{~m}$.

!Plagiochila asplenioides (L. emend. Taylor) Dumort. - [Fe87, 49 $\left.51^{\prime} 26^{\prime \prime} \mathrm{N} / 21^{\circ} 03^{\prime} 01^{\prime \prime E}\right]$ Lubaszowa, near road to the monastery, wayside slope, $260 \mathrm{~m}$; [Fe97, 49 46'41"N/20 58'45"E] Ciężkowice, 'Wodospad' nature monument, on ground in Abies alba forest, $300 \mathrm{~m}$; [Fe88, 49 50'06"N/21 $05^{\circ} 31^{\prime \prime E}$ ] Jodłówka Tuchowska, between Ratówki and Kobyli Dział settlements, wet slope in Abies alba forest, $368 \mathrm{~m}$; [Ge08, 49 $43^{\prime} 04^{\prime N} / 21^{\circ} 05^{\prime} 20^{\prime \prime E] ~ M o s z c z e n i c a, ~ ' K s i e ̨ z ̇ y ~ L a s ' ~ f o r e s t, ~ f o r e s t ~ f l o o r ~ i n ~ A b i e s ~ a l b a ~ f o r e s t, ~}$ $349 \mathrm{~m}$.

Plagiochila porelloides (Torrey ex Nees) Lindenb. - [Ge08, 49 43'04"N/21 $\left.05^{\circ} 20^{\prime \prime} \mathrm{E}\right]$ Moszczenica, 'Księży Las' forest, stream bed in Abies alba forest, $349 \mathrm{~m}$.

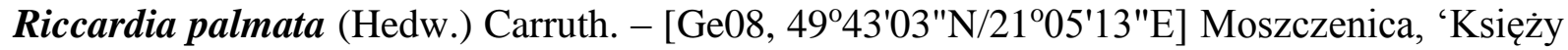
Las' forest, rotten wood of silver fir in Abies alba forest, $365 \mathrm{~m}$.

Riccia glauca L. - [Fe97, 49 47'50"N/21 $\left.{ }^{\circ} 00^{\prime} 05^{\prime \prime E}\right]$ Przedmieście Wielkie, stubble-field, $343 \mathrm{~m}$;

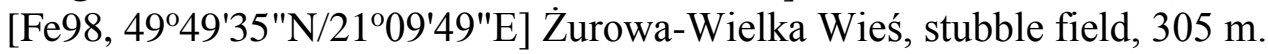

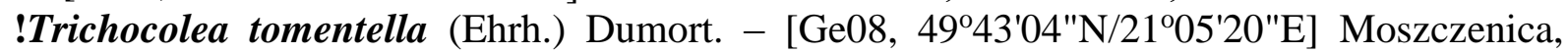
'Księży Las' forest, mineral soil, stream bed in Abies alba forest, $349 \mathrm{~m}$.

\section{MOSSES / BRYOPHYTA}

Amblystegium serpens (Hedw.) Schimp. - [Fe87, 49 51'26"N/21 03'01"E] Lubaszowa, near road to the monastery, wall, c. spor., $260 \mathrm{~m}$; [Fe88, 49 $52^{\prime} 53^{\prime \prime N} / 21^{\circ} 05^{\prime} 46 " \mathrm{E}$ ] Bistuszowa, wall, c. spor., $231 \mathrm{~m}$; [Fe88, 49 52'55"N/2112'16"E] Kowalowa, wall, c. spor., $281 \mathrm{~m}$; [Fe97, 49 46'32"N/20'59'31"E] Ostrusza, abandoned quarry, stone, $266 \mathrm{~m}$; [Ge08, 49 $\left.43^{\prime} 09^{\prime \prime N} / 21^{\circ} 05^{\prime} 24^{\prime \prime E}\right]$ Moszczenica, 'Księży Las' forest, rotten wood of silver fir in Abies alba forest, $340 \mathrm{~m}$.

:Anomodon attenuatus (Hedw.) Huebener - [Fe87, 49 51 '26"N/21 $\left.03^{\circ} 01^{\prime \prime E}\right]$ Lubaszowa, stone wayside slope in deciduous forest near the road to the monastery, $260 \mathrm{~m}$.

Atrichum undulatum (Hedw.) P.Beauv. - [Fe87, 49 51'26"N/21 $03^{\circ}$ '01"E] Lubaszowa, near road to the monastery, wayside, c. spor., $260 \mathrm{~m}$; [Fe88, 49 $\left.52^{\prime} 55^{\prime \prime N} / 21^{\circ} 12^{\prime} 16 " \mathrm{E}\right]$ Kowalowa, slope, $281 \mathrm{~m}$; [Fe89, 49 51'16"N/21'16'28"E] Wisowa, mixed forest, c. spor., $384 \mathrm{~m}$; [Fe88, 49 50'39"N/2105'46"E] Jodłówka Tuchowska-Ratówki, near mountain hut, c. spor., 498 m;

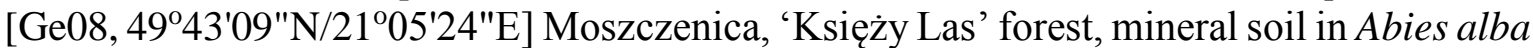
forest, $340 \mathrm{~m}$.

Barbula unguiculata Hedw. - [Ff90, 49 48'13"N/21'22'28"E] Brzyska-Strebikowa, gravelly soil, wayside, c. spor., $398 \mathrm{~m}$.

Brachytheciastrum velutinum (Hedw.) Ignatov \& Huttunen - [Fe87, 49 51'26"N/21 $\left.03^{\circ} 01^{\prime \prime} \mathrm{E}\right]$ Lubaszowa, near road to the monastery, bark of trees, c. spor., $260 \mathrm{~m}$; [Fe89,

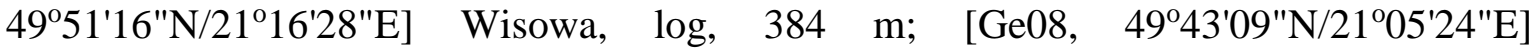
Moszczenica, 'Księży Las' forest, mineral soil in Abies alba forest, $340 \mathrm{~m}$.

Brachythecium albicans (Hedw.) Schimp. - [Fe97, 49 46'32"N/2059'31"E] Ostrusza, abandoned quarry, mineral soil, $266 \mathrm{~m}$.

Brachythecium rivulare Schimp. - [Fe88, 49 50'06"N/2105'31"E] Jodłówka Tuchowska, between Ratówki and Kobyli Dział settlements, seepage in Abies alba forest, c. spor., 368 m; [Ge08, 49 43'09"N/2105'24"E] Moszczenica, 'Księży Las' forest, mineral soil, stream bed in Abies alba forest, $340 \mathrm{~m}$.

Brachythecium rutabulum (Hedw.) Schimp. - [Fe87, 49 51'26"N/21 $\left.03^{\circ} 01^{\prime \prime E}\right]$ Lubaszowa, near road to the monastery, wayside, $260 \mathrm{~m}$; [Fe88, 49 $\left.52^{\prime} 53^{\prime \prime N} / 21^{\circ} 05^{\prime} 46 " \mathrm{E}\right]$ Bistuszowa, 
wayside, $231 \mathrm{~m}$; [Fe89, 49 $\left.51^{\prime} 16^{\prime \prime N} / 21^{\circ} 16^{\prime 2} 28^{\prime \prime E}\right]$ Wisowa, soil in thicket, c. spor., $384 \mathrm{~m}$;

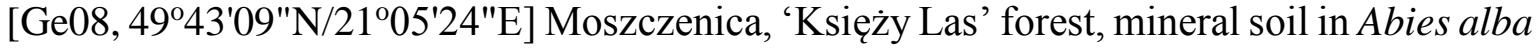
forest, $340 \mathrm{~m}$.

Brachythecium salebrosum (Hoffm. ex F.Weber \& D.Mohr) Schimp. - [Fe87, 49 $51^{\prime} 26^{\prime \prime} \mathrm{N} / 21^{\circ} 03^{\prime} 01^{\prime \prime E}$ ] Lubaszowa, near road to the monastery, wall, c. spor., $260 \mathrm{~m}$;

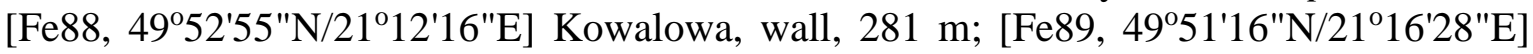

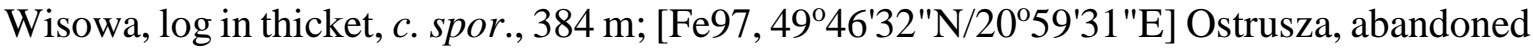
quarry, mineral soil, $266 \mathrm{~m}$; [Fe88, 49 $\left.50^{\circ} 05^{\prime \prime N} / 21^{\circ} 05^{\prime} 33^{\prime \prime E}\right]$ Jodłówka Tuchowska, between Ratówki and Kobyli Dział settlements, stump in Abies alba forest, 383 m; [Ge08, 49 43'03"N/21 $05^{\circ} 13^{\prime \prime E}$ ] Moszczenica, 'Księży Las' forest, rotten wood in Abies alba forest, $365 \mathrm{~m}$.

Bryum argenteum Hedw. - [Fe87, 49 $\left.51^{\prime} 26^{\prime \prime N} / 21^{\circ} 03^{\prime} 01^{\prime \prime} \mathrm{E}\right]$ Lubaszowa, near road to the

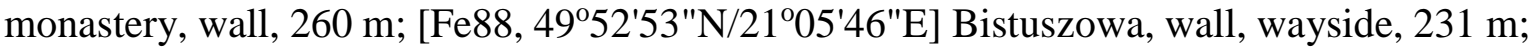
[Fe88, 49 $52^{\prime} 55^{\prime \prime N} / 21^{\circ} 12^{\prime} 16^{\prime E}$ ] Kowalowa, wayside, c.spor., $281 \mathrm{~m}$; [Fe97, 49 46'32"N 12059'31"E] Ostrusza, abandoned quarry, mineral soil, $266 \mathrm{~m}$; [Fe98, 4949'35"N $\left.121^{\circ} 09^{\prime} 49^{\prime \prime E}\right]$ Żurowa-Wielka Wieś, stubble field, $305 \mathrm{~m}$.

Bryum rubens Mitt. - [Ge08, 49 $\left.43^{\prime} 03^{\prime \prime N} / 21^{\circ} 05^{\prime} 13 " \mathrm{E}\right]$ Moszczenica, 'Księży Las' forest, mineral soil, dry ditch on margin of Abies alba forest, c.gem., $365 \mathrm{~m}$.

Callicladium haldanianum (Grev.) H.A.Crum - [Fe88, 49 $\left.50^{\prime} 05^{\prime \prime N} / 21^{\circ} 05^{\prime} 33^{\prime \prime E}\right]$ Jodłówka Tuchowska, between Ratówki and Kobyli Dział settlements, log in Abies alba forest, $c$. spor., $383 \mathrm{~m}$; [Ff90, 49 $48^{\prime} 13^{\prime \prime} \mathrm{N} / 21^{\circ} 22^{\prime} 28^{\prime \prime E}$ ] Brzyska-Strebikowa, stump in Abies alba

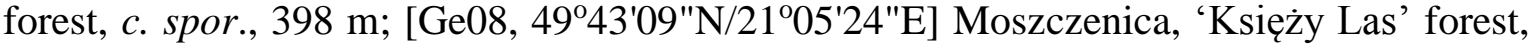
rotten wood of silver fir in Abies alba forest, $340 \mathrm{~m}$.

!Calliergonella cuspidata (Hedw.) Loeske - [Fe87, 49 $\left.51^{\prime} 26^{\prime \prime} \mathrm{N} / 21^{\circ} 03^{\prime} 01^{\prime \prime E}\right]$ Lubaszowa, near road to the monastery, ditch, $255 \mathrm{~m}$; [Fe88, 49 $\left.52^{\prime} 53^{\prime \prime N} / 21^{\circ} 05^{\prime} 46^{\prime \prime E}\right]$ Bistuszowa, ditch, 231 $\mathrm{m}$; $\left[\mathrm{Fe} 89,49^{\circ} 51^{\prime} 16^{\prime \prime} \mathrm{N} / 21^{\circ} 16^{\prime} 28^{\prime \prime E}\right]$ Wisowa, wet place, $384 \mathrm{~m}$; [Fe88, 49 $\left.50^{\prime} 36^{\prime \prime N} / 21^{\circ} 05^{\prime} 55^{\prime \prime E}\right]$ Jodłówka Tuchowska-Ratówki, meadow near mountain hut, 492 $\mathrm{m} ; \quad\left[\mathrm{Fe} 88,49^{\circ} 52^{\prime} 55^{\prime \prime} \mathrm{N} / 21^{\circ} 12^{\prime} 16 " \mathrm{E}\right]$ Kowalowa, wet meadow, $281 \mathrm{~m} ; \quad[\mathrm{Ge} 08$, 49 43'03"N/21 $05^{\circ} 13^{\prime \prime E}$ ] Moszczenica, 'Księży Las' forest, moist mineral soil in Abies alba forest, $365 \mathrm{~m}$.

!Campylopus flexuosus (Hedw.) Brid. - [Fe97, 49 46'43"N/2058'09"E] 'Skamieniałe Miasto' nature reserve, mineral soil in Pinus sylvestris forest, $320 \mathrm{~m}$; [Fe98, 49 $49^{\prime} 26^{\prime \prime N} / 21^{\circ} 10^{\prime} 21^{\prime \prime E}$ ] Żurowa, 'Borówka' sandstone outcrop, sandstone and path in Pinus sylvestris forest, $339 \mathrm{~m}$; [Fe88, 49 $\left.50^{\prime} 39^{\prime \prime N} / 21^{\circ} 05^{\prime} 41^{\prime \prime E}\right]$ Jodłówka Tuchowska-Ratówki, mineral soil near sandstone outcrop near mountain hut, $501 \mathrm{~m}$.

Campylopus introflexus (Hedw.) Brid. - [Fe97, 49 46'32"N/20'59'31"E] Ostrusza, abandoned quarry, mineral soil and sandstone, $266 \mathrm{~m}$; [Ff90, 49 48'13"N/21'22'28"E] BrzyskaStrebikowa, on ground in Abies alba forest, 398 m; [Ff90, 4948'19"N/21 $22^{\circ} 03^{\prime \prime E}$ ] BrzyskaZakościele Góry, wayside in Pinus sylvestris forest, $444 \mathrm{~m}$.

Ceratodon purpureus (Hedw.) Brid. - [Fe87, 49 51'26"N/21 $\left.03^{\circ} 01^{\prime \prime E}\right]$ Lubaszowa, near road to the monastery, wall, waste place, c. spor., $260 \mathrm{~m}$; [Ff90, 49 $48^{\prime} 13^{\prime \prime N} / 21^{\circ} 22^{\prime 2} 28^{\prime \prime E}$ ]

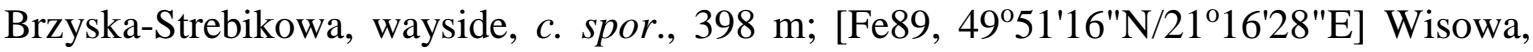
waste place $384 \mathrm{~m}$; [Fe97, 49 $46^{\prime} 32^{\prime \prime N} / 20^{\circ} 59^{\prime} 31^{\prime \prime E}$ ] Ostrusza, abandoned quarry, mineral soil, $266 \mathrm{~m}$; [Fe98, 49 49'35"N/2109'49"E] Żurowa-Wielka Wieś, stubble-field, $305 \mathrm{~m}$; [Ge08, 49 43'03"N/21 $\left.05^{\circ} 13^{\prime \prime E}\right]$ Moszczenica, 'Księży Las' forest, dry ditch on margin of Abies alba forest, $365 \mathrm{~m}$.

Cirriphyllum piliferum (Hedw.) Grout - [Fe87, 49 51'26"N/21 $\left.03^{\prime} 01^{\prime \prime E}\right]$ Lubaszowa, near road to the monastery, slope near stream, $255 \mathrm{~m}$; [Ge08, 4943'09"N/2105'24"E] Moszczenica, 'Księży Las' forest, mineral soil in Abies alba forest, 340 m. 
!Climacium dendroides (Hedw.) F.Weber \& D.Mohr - [Fe88, 49 $\left.52^{\prime} 55^{\prime \prime N} / 21^{\circ} 12^{\prime} 16^{\prime \prime E}\right]$ Kowalowa, bank of meadow, wayside, $281 \mathrm{~m}$.

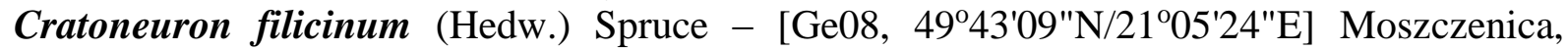
'Księży Las' forest, sandstone in stream bed in Abies alba forest, $340 \mathrm{~m}$.

Dichodontium pellucidum (Hedw.) Schimp. - [Fe87, 49 51'26"N/21 $\left.{ }^{\circ} 03^{\prime} 01^{\prime \prime E}\right]$ Lubaszowa, near road to the monastery, boulders in stream, $255 \mathrm{~m}$; [Ge08, 49 $\left.43^{\prime} 04^{\prime \prime N} / 21^{\circ} 05^{\prime} 20^{\prime \prime} \mathrm{E}\right]$ Moszczenica, 'Księży Las' forest, sandstone, stream bed in Abies alba forest, $349 \mathrm{~m}$.

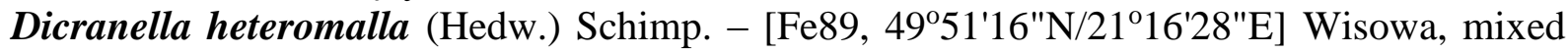
forest, 384 m; [Fe88, 4950'06"N/21 ${ }^{\circ} 05^{\prime} 31^{\prime \prime E}$ ] Jodłówka Tuchowska, between Ratówki and Kobyli Dział settlements, wet slope in Abies alba forest, c. spor., $368 \mathrm{~m}$; [Ge08, 49 $\left.43^{\prime} 04^{\prime \prime N} / 21^{\circ} 05^{\prime} 20^{\prime \prime E}\right]$ Moszczenica, 'Księży Las' forest, mineral soil in Abies alba forest, c.spor., $349 \mathrm{~m}$.

Dicranella rufescens (Dicks.) Schimp. - [Fe87, 49 51'29"N/21 $03^{\circ}$ '02"E] Lubaszowa, near road to the monastery, landslide, c. spor., $255 \mathrm{~m}$; [Fe88, 49 $\left.50^{\prime} 42^{\prime \prime N} / 21^{\circ} 08^{\prime} 50 " \mathrm{E}\right]$ ŻurowaPodlesie Drugie, stubble field, $469 \mathrm{~m}$.

Dicranella varia (Hedw.) Schimp. - [Ff90, 4948'13"N/2122'28"E] Brzyska-Strebikowa, gravelly soil, wayside, c. spor., $398 \mathrm{~m}$.

Dicranodontium denudatum (Brid.) E.Britton - [Fe98, 4949'26"N/21 $\left.{ }^{\circ} 10^{\prime} 25^{\prime \prime E}\right]$ Żurowa, 'Borówka' sandstone outcrop, sandstone, $359 \mathrm{~m}$.

!Dicranum scoparium Hedw. - Humus soil, rotten wood, bark, sandstone. [Fe88, 49 50'49"N/21 08'07"E] Mt Pasia, south-eastern slope, $504 \mathrm{~m}$; [Fe88, 49 $50^{\prime} 40^{\prime \prime N} / 21^{\circ} 08^{\prime} 51^{\prime \prime E}$ ] Żurowa-Podlesie Drugie, 472 m; [Fe97, 494' $\left.41^{\circ} \mathrm{N} / 20^{\circ} 57^{\prime} 55^{\prime \prime E}\right]$ Ciężkowice, 'Skamieniałe Miasto' nature reserve, 302 m; [Fe97, 4946'41"N/2058'47"E] Ciężkowice, 'Wodospad' nature monument, $311 \mathrm{~m}$; [Fe89, 4951'16"N/21º'16'28"E]

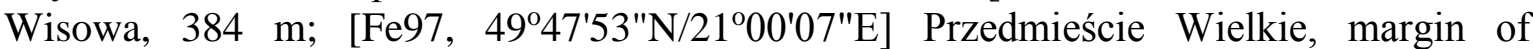
coniferous forest, $332 \mathrm{~m}$; [Fe98, 49 49'24"N/2110'16"E] Żurowa, 'Borówka' sandstone outcrop, wayside in mixed forest, $332 \mathrm{~m}$; [Fe88, 49 $50^{\prime} 39^{\prime \prime N} / 21^{\circ} 05^{\prime} 41^{\prime \prime E}$ ] Jodłówka Tuchowska-Ratówki, near mountain hut, $501 \mathrm{~m}$; [Ff90, 4948'13"N/2122'28"E] Brzyska-

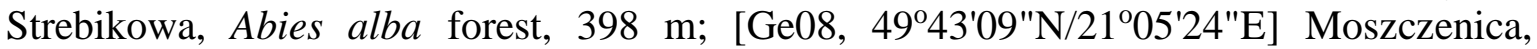
'Księży Las' forest, forest floor in Abies alba forest, $340 \mathrm{~m}$.

Didymodon rigidulus Hedw. - [Fe87, 49 $\left.51^{\prime 2} 26^{\prime \prime N} / 21^{\circ} 03^{\prime} 01^{\prime \prime} \mathrm{E}\right]$ Lubaszowa, near road to the

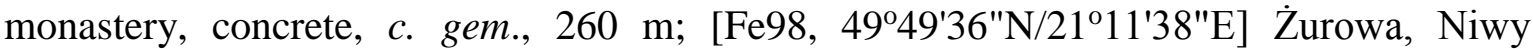
settlement, on the concrete tile on the roof of barn, c. gem., $280 \mathrm{~m}$.

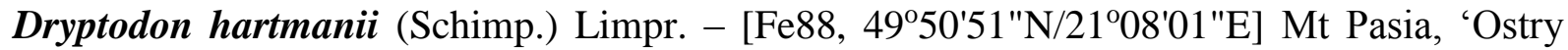
Kamień' sandstone outcrop, sandstone, c. gem., 526 m.

!Eurhynchium angustirete (Broth.) T.J.Kop. - Soil in forests and thickets, rotten wood. [Fe87, $\left.49^{\circ} 51^{\prime} 26^{\prime \prime N} / 21^{\circ} 03^{\prime} 01^{\prime \prime E}\right]$ Lubaszowa, near road to the monastery, $260 \mathrm{~m}$; [Fe97, 49 46'41"N/20 58'44"E] Ciężkowice, 'Wodospad' nature monument, Abies alba forest, 301 m; [Ff90, 4948'13"N/2122'28"E] Brzyska-Strebikowa, Abies alba forest, 398 m; [Ge08, 49 43'09"N/2105'24"E] Moszczenica, 'Księży Las' forest, Abies alba forest, $340 \mathrm{~m}$.

Fissidens bryoides Hedw. - [Ff90, 49 48'13"N/21 222'28"E] Brzyska-Strebikowa, wayside in mixed forest, c. spor., 398 m; [Ge08, 49 43'09"N/2105'24"E] Moszczenica, 'Księży Las' forest, mineral soil in Abies alba forest, c.spor., $340 \mathrm{~m}$.

Fissidens taxifolius Hedw. - [Fe87, 49 51'26"N/21 $\left.03^{\prime} 01^{\prime \prime} \mathrm{E}\right]$ Lubaszowa, near road to the

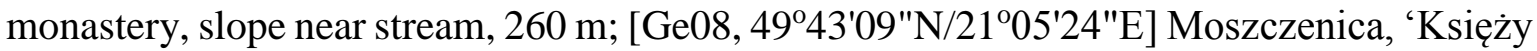
Las' forest, wet mineral soil in Abies alba forest, $340 \mathrm{~m}$.

Fissidens pusillus (Wilson) Milde - [Fe87, 49 51'26"N/21 $03^{\circ}$ '01 "E] Lubaszowa, near road to the monastery, boulder in stream, c. spor., $260 \mathrm{~m}$.

Funaria hygrometrica Hedw. - [Fe88, 49 $\left.52^{\prime} 53^{\prime \prime N} / 21^{\circ} 05^{\prime} 46^{\prime E}\right]$ Bistuszowa, wayside, c. spor., $231 \mathrm{~m}$. 
Herzogiella seligeri (Brid.) Z.Iwats. - [Fe89, 49 $\left.51^{\prime} 16^{\prime \prime} \mathrm{N} / 21^{\circ} 16^{\prime} 28^{\prime \prime E}\right]$ Wisowa, rotten wood in mixed forest, $384 \mathrm{~m}$; [Fe88, 49 $50^{\prime} 05^{\prime N} / 21^{\circ} 05^{\prime} 33^{\prime \prime E}$ ] Jodłówka Tuchowska, between Ratówki and Kobyli Dział settlements, stump in Abies alba forest, $383 \mathrm{~m}$; [Ge08, 49 43'09"N/21 05'24"E] Moszczenica, 'Księży Las' forest, rotten wood of silver fir in Abies alba forest, c.spor., $340 \mathrm{~m}$.

Hygrohypnum luridum (Hedw.) Jenn. - [Fe87, 49 51'26"N/21 $\left.03^{\circ} 01^{\prime \prime E}\right]$ Lubaszowa, near road to the monastery, boulders in stream and concrete, c. spor., 255-260 m; [Ge08, 49 43'09"N/21 05'24"E] Moszczenica, 'Księży Las' forest, sandstone, the stream bed in Abies alba forest, c.spor., $340 \mathrm{~m}$.

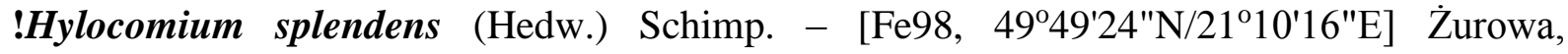
'Borówka' sandstone outcrop, wayside in mixed forest, $332 \mathrm{~m}$; $[\mathrm{Fe} 88$, 49 50'05"N/2105'33"E] Jodłówka Tuchowska, between Ratówki and Kobyli Dział settlements, on ground in Abies alba forest, $383 \mathrm{~m}$.

Hypnum cupressiforme Hedw. - Bark, soil and rotten wood. [Fe87, 49 $\left.51^{\prime} 26^{\prime \prime} \mathrm{N} / 21^{\circ} 03^{\prime} 01^{\prime \prime} \mathrm{E}\right]$ Lubaszowa, near road to the monastery, $260 \mathrm{~m}$; [Fe89, 49 $51^{\prime} 16^{\prime \prime N} / 21^{\circ} 16^{\prime} 28$ "E] Wisowa, $384 \mathrm{~m}$; [Fe97, 4946'32"N/2059'31"E] Ostrusza, abandoned quarry, $266 \mathrm{~m}$; [Fe88, 49 $\left.50^{\prime} 05^{\prime \prime N} / 21^{\circ} 05^{\prime} 33^{\prime \prime E}\right]$ Jodłówka Tuchowska, between Ratówki and Kobyli Dział

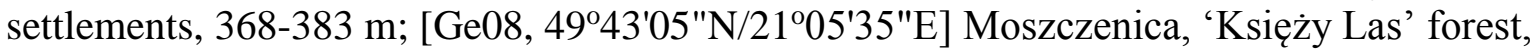
Abies alba forest, $344 \mathrm{~m}$.

Hypnum lindbergii Mitt. - [Ff90, 49 48'13"N/21 $\left.22^{\prime} 28^{\prime \prime} \mathrm{E}\right]$ Brzyska-Strebikowa, wayside, $c$. spor., $398 \mathrm{~m}$; [Fe97, 49046'32"N/2059'31"E] Ostrusza, abandoned quarry, mineral soil, 266 m; [Ge08, 4943'03"N/21 05'13"E] Moszczenica, 'Księży Las' forest, mineral soil in Abies alba forest, $365 \mathrm{~m}$.

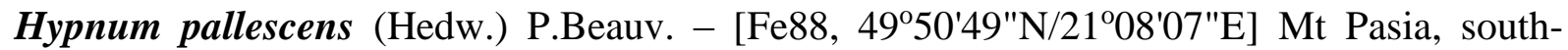
eastern slope, Fagus sylvatica stump, c. spor., $504 \mathrm{~m}$; [Fe97, 49 46'41"N/2057'55"E] 'Skamieniałe Miasto' nature reserve, sandstone, c. spor., $302 \mathrm{~m}$; [Fe88, 49 $50^{\prime} 39^{\prime N} / 21^{\circ} 05^{\prime} 46^{\prime \prime E] ~ J o d ł o ́ w k a ~ T u c h o w s k a-R a t o ́ w k i, ~ b a r k ~ o f ~ F a g u s ~ s y l v a t i c a ~ i n ~ b e e c h ~}$ forest near mountain hut, c. spor., $498 \mathrm{~m}$; [Ge08, 49 43'09"N/21 $\left.05^{\circ} 24^{\prime \prime E}\right]$ Moszczenica, 'Księży Las' forest, bark of Quercus robur in Abies alba forest, $340 \mathrm{~m}$.

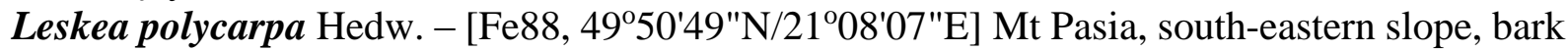

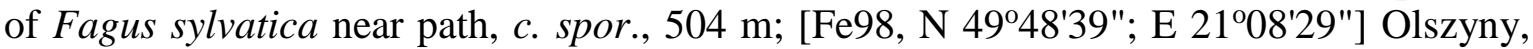
bark of wayside Fraxinus excelsior, c. spor., $290 \mathrm{~m}$; [Ff90, 4949'14"N/21²3'25"E] Brzyska, bark of Fraxinus excelsior in old park near the castle, $241 \mathrm{~m}$.

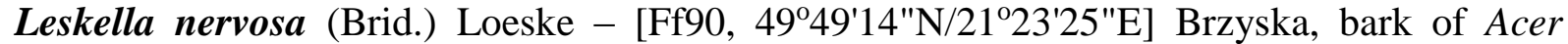
platanoides in old park near the castle, $241 \mathrm{~m}$.

!Leucobryum glaucum (Hedw.) Ångstr. - [Fe97, 4946'43"N/2058'09"E] Ciężkowice, 'Skamieniałe Miasto' nature reserve, soil in Pinus sylvestris forest, $320 \mathrm{~m}$; [Fe97, 49 46'41"N/20 58'47"E] Ciężkowice, 'Wodospad' nature monument, on ground in Abies alba forest, $297 \mathrm{~m}$; [Fe98, 49 49'23"N/21 $\left.{ }^{\circ} 10^{\prime} 25^{\prime \prime} \mathrm{E}\right]$ Żurowa, 'Borówka' sandstone outcrop, wayside in Pinus sylvestris forest, $349 \mathrm{~m}$; [Fe88, 4950'06"N/2105'36"E] Jodłówka Tuchowska, between Ratówki and Kobyli Dział settlements, on ground in Abies alba forest, $389 \mathrm{~m}$; [Ge08, 49 $\left.43^{\prime} 05^{\prime \prime N} / 21^{\circ} 05^{\prime} 35^{\prime \prime E}\right]$ Moszczenica, 'Księży Las' forest, forest floor in Abies alba forest, $344 \mathrm{~m}$.

Mnium hornum Hedw. - [Fe87, 49 51'26"N/21 $\left.{ }^{\circ} 03^{\prime} 01^{\prime \prime E}\right]$ Lubaszowa, near road to the

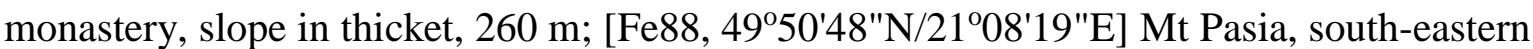
slope, on soil near path, $473 \mathrm{~m}$; [Fe97, 4946'41"N/2057'55"E] 'Skamieniałe Miasto' nature reserve, sandstone, $302 \mathrm{~m}$; [Fe97, 4946'41"N/20'58'47"E] Ciężkowice, 'Wodospad' nature monument, on ground in Abies alba forest, $311 \mathrm{~m}$; [Fe98, 49 $\left.49^{\prime} 26^{\prime \prime N} / 21^{\circ} 10^{\prime} 25^{\prime \prime E}\right]$ Żurowa, 'Borówka' sandstone outcrop, sandstone, 359 m; [Fe88, 49 50'05"N/21 $05^{\circ} 33^{\circ}$ "E] Jodłówka Tuchowska, between Ratówki and Kobyli Dział settlements, log in Abies alba forest, 383 
m; [Ge08, 49 43'04"N/2105'20"E] Moszczenica, 'Księży Las' forest, forest floor in Abies alba forest, $349 \mathrm{~m}$.

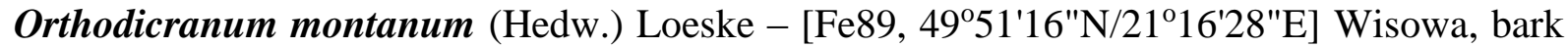
of deciduous trees, $384 \mathrm{~m}$; [Fe97, 49 47'53"N/21 $\left.{ }^{\circ} 00^{\prime} 07^{\prime \prime E}\right]$ Przedmieście Wielkie, stump in coniferous forest, $332 \mathrm{~m}$; [Fe88, 49 50 '05"N/2105'33"E] Jodłówka Tuchowska, between Ratówki and Kobyli Dział settlements, stump in Abies alba forest, $383 \mathrm{~m}$; [Ge08, 49 43'09"N/21 05'24"E] Moszczenica, 'Księży Las' forest, rotten wood of silver fir in Abies alba forest, $340 \mathrm{~m}$.

Orthotrichum anomalum Hedw. - [Fe98, N 4948'39"; E 21 08'29"] Olszyny, bark of wayside Fraxinus excelsior, c. spor., $290 \mathrm{~m}$; [Ff90, 49 49'14"N/2123'25"E] Brzyska, bark of Fraxinus excelsior in old park near the castle, c. spor., $241 \mathrm{~m}$.

Orthotrichum affine Schrad. ex Brid. - [Ge08, 4943'01"N/21 05'26"E] Moszczenica, 'Księży Las' forest, bark of Fraxinus excelsior in Abies alba forest, c.spor., $346 \mathrm{~m}$.

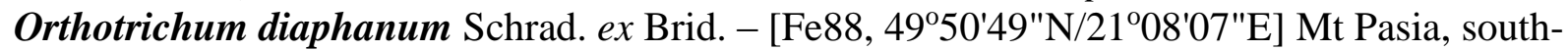
eastern slope, bark of Fagus sylvatica near path, c. spor., 504 m; [Fe98, N 4948'39"; E $21^{\circ} 08^{\prime 29 "] ~ O l s z y n y, ~ b a r k ~ o f ~ w a y s i d e ~ F r a x i n u s ~ e x c e l s i o r, ~ c . ~ s p o r ., ~} 290 \mathrm{~m}$.

[R]Orthotrichum patens Bruch ex Brid. (Fig. 5) - [Fe88, 49 50'49"N/21 08'07"E] Mt Pasia, south-eastern slope, bark of Fagus sylvatica near path, c. spor., 504 m; [Ff90, 49 $\left.49^{\prime} 14^{\prime \prime} \mathrm{N} / 21^{\circ} 23^{\prime} 25^{\prime \prime} \mathrm{E}\right]$ Brzyska, bark of Acer platanoides in old park near the castle, $c$. spor., $241 \mathrm{~m}$.

Orthotrichum pumilum Sw. - [Fe87, 49 $\left.51^{\prime} 26 " \mathrm{~N} / 21^{\circ} 03^{\prime} 01^{\prime \prime E}\right]$ Lubaszowa, near road to the monastery, concrete, c. spor., 255 m; [Ff90, 4949'14"N/2123'25"E] Brzyska, bark of Fraxinus excelsior in old park near the castle, c. spor., $241 \mathrm{~m}$.

Orthotrichum speciosum Nees - [Ff90, 49 $\left.49^{\circ} 14^{\prime \prime N} / 21^{\circ} 23^{\prime} 25^{\prime \prime E}\right]$ Brzyska, bark of Fraxinus excelsior in old park near the castle, c. spor., $241 \mathrm{~m}$; [Fe88, 49 $\left.52^{\prime} 53^{\prime \prime N} / 21^{\circ} 05^{\prime} 46^{\prime \prime} \mathrm{E}\right]$ Bistuszowa, bark of Tilia cordata, c. spor., $234 \mathrm{~m}$; [Ge08, 49 $43^{\prime} 01^{\prime \prime N} / 21^{\circ} 05^{\prime} 26^{\prime \prime E}$ ] Moszczenica, 'Księży Las' forest, bark of Fraxinus excelsior in Abies alba forest, c.spor., $346 \mathrm{~m}$.

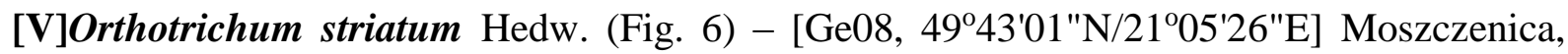
'Księży Las' forest, bark of Fraxinus excelsior in Abies alba forest, c.spor., $346 \mathrm{~m}$.

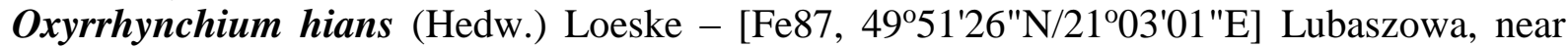
road to the monastery, mineral soil, $260 \mathrm{~m}$; [Fe97, 49 46'32"N/20'59'31"E] Ostrusza, abandoned quarry, mineral soil, $266 \mathrm{~m}$; [Ge08, 49 43'09"N/2105'24"E] Moszczenica, 'Księży Las' forest, mineral soil in Abies alba forest, $340 \mathrm{~m}$.

Paraleucobryum longifolium (Ehrh. ex Hedw.) Loeske - [Fe88, 49 50'51"N/21 08'01"E] Mt Pasia, 'Ostry Kamień' sandstone outcrop, sandstone, 526 m; [Fe97, 4946'39"N/2057'55"E] 'Skamieniałe Miasto' nature reserve, sandstone, $302 \mathrm{~m}$.

Plagiomnium affine (Blandow ex Funck) T.J.Kop. - On ground in forests and thickets. [Fe89,

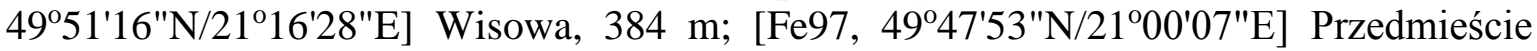
Wielkie, margin of coniferous forest, $332 \mathrm{~m}$; [Fe98, 49 $49^{\prime} 24^{\prime \prime N} / 21^{\circ} 10^{\prime} 16^{\prime \prime E}$ ] Żurowa, 'Borówka' sandstone outcrop, wayside, $332 \mathrm{~m}$; [Fe88, 4950'06"N/21055'31"E] Jodłówka Tuchowska, between Ratówki and Kobyli Dział settlements, Abies alba forest, c. spor., 368 m; [Ge08, 4943'09"N/21 ํ5'24"E] Moszczenica, 'Księży Las' forest, Abies alba forest, 340 $\mathrm{m}$.

Plagiomnium cuspidatum (Hedw.) T.J.Kop. - [Ge08, 49 43'04"N/21 $\left.05^{\prime} 20 " \mathrm{E}\right]$ Moszczenica, 'Księży Las' forest, forest floor in Abies alba forest, $349 \mathrm{~m}$.

Plagiomnium undulatum (Hedw.) T.J.Kop. - [Fe87, 49 51'26"N/21 $\left.03^{\circ} 01^{\prime \prime E}\right]$ Lubaszowa, near road to the monastery, bank of stream, $260 \mathrm{~m}$; [Fe88, 49 $52^{\prime} 53^{\prime \prime N} / 21^{\circ} 05^{\prime} 46^{\prime \prime} \mathrm{E}$ ]

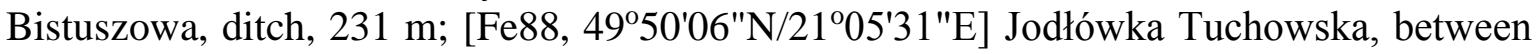
Ratówki and Kobyli Dział settlements, springs in Abies alba forest, $368 \mathrm{~m}$; [Ge08, 
49 43'04"N/21 05'20"E] Moszczenica, 'Księży Las' forest, forest floor in Abies alba forest, $349 \mathrm{~m}$.

Plagiothecium cavifolium (Brid.) Z.Iwats. - [Fe87, 49 $\left.51^{\prime} 26^{\prime \prime} \mathrm{N} / 21^{\circ} 03^{\prime} 01^{\prime \prime E}\right]$ Lubaszowa, near road to the monastery, mineral soil in thicket, $260 \mathrm{~m}$.

Plagiothecium curvifolium Schlieph. ex Limpr. - [Fe89, 49 $\left.51^{\prime} 16^{\prime \prime N} / 21^{\circ} 16^{\prime} 28^{\prime \prime E}\right]$ Wisowa, 384 m; [Fe88, 49 50'06"N/21 $\left.05^{\prime} 31^{\prime \prime E}\right]$ Jodłówka Tuchowska, between Ratówki and Kobyli Dział settlements, on ground and rotten wood in Abies alba forest, c. spor., 368-383 m;

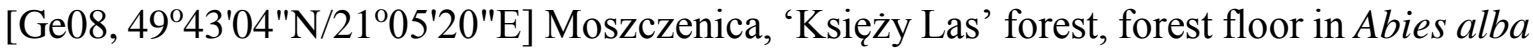
forest, $349 \mathrm{~m}$.

Plagiothecium denticulatum (Hedw.) Schimp. - [Ge08, 49 43'05"N/21 $\left.{ }^{\circ} 05^{\prime} 35^{\prime \prime E}\right]$ Moszczenica, 'Księży Las' forest, forest floor in Abies alba forest, c.spor., $344 \mathrm{~m}$.

Plagiothecium laetum Schimp. - [Ge08, 4943'03"N/2105'13"E] Moszczenica, 'Księży Las' forest, bark of Quercus robur in Abies alba forest, $365 \mathrm{~m}$.

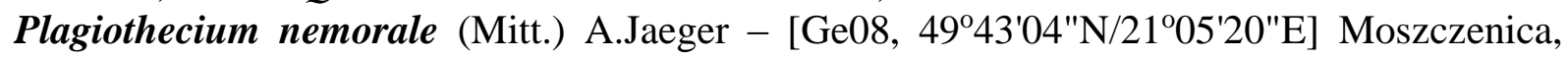
'Księży Las' forest, forest floor in Abies alba forest, $349 \mathrm{~m}$.

!Pleurozium schreberi (Willd. ex Brid.) Mitt. - On ground in forests and thickets, grasslands. [Fe88, 49 $\left.50^{\prime} 40 " \mathrm{~N} / 21^{\circ} 08^{\prime} 51^{\prime \prime E}\right]$ Żurowa-Podlesie Drugie, $472 \mathrm{~m}$; [Fe89, 49 $51^{\prime} 16^{\prime N} / 21^{\circ} 16^{\prime 2} 28^{\prime E}$ ] Wisowa, $384 \mathrm{~m}$; [Fe97, 4946'39"N/2058'11"E] 'Skamieniałe

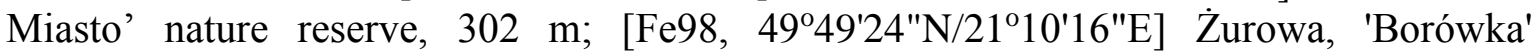

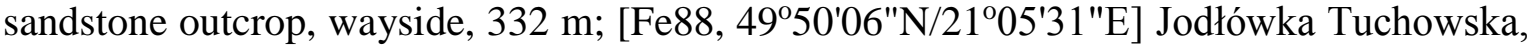
between Ratówki and Kobyli Dział settlements, Abies alba forest, $368 \mathrm{~m}$; [Ff90, 49 48'13"N/2122'28"E] Brzyska-Strebikowa, Abies alba forest, $398 \mathrm{~m}$; [Ge08, 49 43'09"N/21 05'24"E] Moszczenica, 'Księży Las' forest, forest floor in Abies alba forest, $340 \mathrm{~m}$.

Pogonatum urnigerum (Hedw.) P.Beauv. - [Fe97, 4946'32"N/2059'31"E] Ostrusza, abandoned quarry, mineral soil, $266 \mathrm{~m}$.

Pohlia camptotrachela (Renauld \& Cardot) Broth. - [Fe88, 4950'42"N/21 $\left.08^{\circ} 50 " E\right]$ ŻurowaPodlesie Drugie, stubble field, c. gem., $469 \mathrm{~m}$.

Pohlia nutans (Hedw.) Lindb. - On ground in forests and thickets. [Fe89,

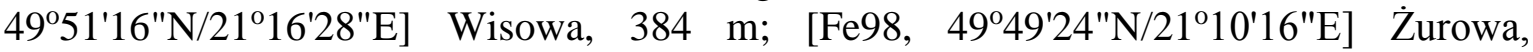
'Borówka' sandstone outcrop, mixed forest, $332 \mathrm{~m}$; [Fe88, 49 $50^{\circ} 05^{\prime \prime N} / 21^{\circ} 05^{\prime} 33^{\prime \prime E}$ ] Jodłówka Tuchowska, between Ratówki and Kobyli Dział settlements, Abies alba forest, 383 m; [Ge08, 4943'09"N/2105'24"E] Moszczenica, 'Księży Las' forest, Abies alba forest, $340 \mathrm{~m}$.

Pohlia wahlenbergii (F.Weber \& D.Mohr) A.L.Andrews - [Ge08, 49 43'09"N/21 $\left.05^{\prime} 24^{\prime \prime E}\right]$ Moszczenica, 'Księży Las' forest, mineral soil in Abies alba forest, $340 \mathrm{~m}$.

Polytrichastrum formosum (Hedw.) G.L.Sm. - On ground in forests and thickets. [Fe87, 49 $\left.51^{\prime} 26^{\prime \prime N} / 21^{\circ} 03^{\prime} 01^{\prime \prime E}\right]$ Lubaszowa, near road to the monastery, $260 \mathrm{~m}$; [Fe89, 49 $51^{\prime} 16^{\prime \prime N} / 21^{\circ} 16^{\prime 2} 28^{\prime E}$ ] Wisowa, $384 \mathrm{~m}$; [Fe97, 4946'39"N/2058'11"E] 'Skamieniałe Miasto' nature reserve, $302 \mathrm{~m}$; [Fe88, 4950'06"N/2105'31"E] Jodłówka Tuchowska, between Ratówki and Kobyli Dział settlements, Abies alba forest, c. spor., 368 m; [Ge08, 49 $\left.43^{\prime} 05^{\prime \prime N} / 21^{\circ} 05^{\prime} 35^{\prime \prime E}\right]$ Moszczenica, 'Księży Las' forest, Abies alba forest, c.spor., 344 m.

Polytrichum piliferum Hedw. - [Fe97, 49²6'32"N/2059'31"E] Ostrusza, abandoned quarry, mineral soil, $266 \mathrm{~m}$.

!Pseudoscleropodium purum (Hedw.) M.Fleisch. ex Broth. - On ground in forests and thickets, grasslands. [Fe87, 49 $51^{\prime} 26^{\prime \prime N} / 21^{\circ} 03^{\prime} 01^{\prime \prime E}$ ] Lubaszowa, near road to the monastery, $260 \mathrm{~m}$; [Fe88, 49 $\left.52^{\prime} 55^{\prime \prime N} / 21^{\circ} 12^{\prime} 16^{\prime \prime E}\right]$ Kowalowa, $281 \mathrm{~m}$; [Fe97, 4946'44"N/20⒌ $\left.58^{\prime} 11^{\prime \prime E}\right]$ Ciężkowice, 'Skamieniałe Miasto' nature reserve, Pinus sylvestris forest, 318 m; [Fe97, 49 46'41"N/20 58'45"E] Ciężkowice, 'Wodospad' nature monument, Abies alba forest, 300 
m; [Fe98, 49 49'24"N/21 $\left.{ }^{\circ} 10^{\prime} 16^{\prime \prime E}\right]$ Żurowa, 'Borówka' sandstone outcrop, mixed forest, 332 m; [Ff90, 4948'13"N/21 '22'28"E] Brzyska-Strebikowa, Abies alba forest, 398 m; [Ge08, 49 $43^{\prime} 05^{\prime N} / 21^{\circ} 05^{\prime} 35^{\prime \prime E] ~ M o s z c z e n i c a, ~ ' K s i e ̨ z ̇ y ~ L a s ' ~ f o r e s t, ~ A b i e s ~ a l b a ~ f o r e s t, ~} 344 \mathrm{~m}$.

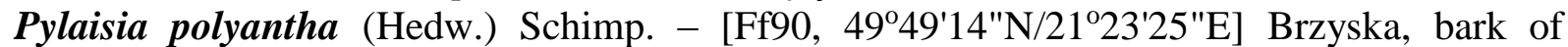
Fraxinus excelsior in old park near the castle, $241 \mathrm{~m}$; [Ge08, 49 $43^{\prime} 01^{\prime \prime N} / 21^{\circ} 05^{\prime} 26^{\prime \prime E}$ ] Moszczenica, 'Księży Las' forest, bark of Fraxinus excelsior in Abies alba forest, c.spor., $346 \mathrm{~m}$.

Rhizomnium punctatum (Hedw.) T.J.Kop. - [Fe88, 4950'06"N/2105'31"E] Jodłówka Tuchowska, between Ratówki and Kobyli Dział settlements, seepage in Abies alba forest, $c$. spor., $368 \mathrm{~m}$; [Ge08, 49 43'09"N/21 05'24"E] Moszczenica, 'Księży Las' forest, rotten wood in Abies alba forest, $340 \mathrm{~m}$.

!Rhytidiadelphus squarrosus (Hedw.) Warnst. - Grasslands, meadows, waste places, on ground in forests and thickets. [Fe88, 49 $\left.52^{\prime} 53^{\prime \prime N} / 21^{\circ} 05^{\prime} 46^{\prime \prime E}\right]$ Bistuszowa, $231 \mathrm{~m}$; [Fe88, 49 $52^{\prime} 55^{\prime \prime N} / 21^{\circ} 12^{\prime} 16^{\prime \prime E}$ ] Kowalowa, $281 \mathrm{~m}$; [Fe88, 49 $\left.50^{\prime} 40 " \mathrm{~N} / 21^{\circ} 08^{\prime} 51^{\prime \prime E}\right]$ ŻurowaPodlesie Drugie, $472 \mathrm{~m}$; [Fe89, 49 $\left.51^{\prime} 16^{\prime \prime N} / 21^{\circ} 16^{\prime} 28^{\prime \prime E}\right]$ Wisowa, $384 \mathrm{~m}$; [Fe97, 49 46'44"N/20 58'11"E] Ciężkowice, 'Skamieniałe Miasto' nature reserve, Pinus sylvestris forest, $318 \mathrm{~m}$; [Fe97, 4946'41"N/20'58'45"E] Ciężkowice, 'Wodospad' nature monument,

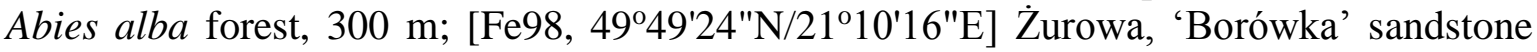
outcrop, mixed forest, $332 \mathrm{~m}$; [Fe88, 49 $\left.50^{\prime} 36^{\prime N} \mathrm{~N} / 21^{\circ} 05^{\prime} 55^{\prime \prime E}\right]$ Jodłówka TuchowskaRatówki, near mountain hut, 492 m; [Ge08, 4943'03"N/21005'13"E] Moszczenica, 'Księży Las' forest, Abies alba forest, $365 \mathrm{~m}$.

Rhytidiadelphus subpinnatus (Lindb.) T.J.Kop. - [Ge08, 49 43'04"N/21 $\left.{ }^{\circ} 05^{\prime} 20^{\prime \prime E}\right]$ Moszczenica, 'Księży Las' forest, moist mineral soil, stream bed in Abies alba forest, 349 m.

Rosulabryum moravicum (Podp.) Ochyra \& Stebel - [Fe89, 49 $\left.51^{\prime} 16^{\prime \prime} \mathrm{N} / 21^{\circ} 16^{\prime} 28^{\prime \prime E}\right]$ Wisowa,

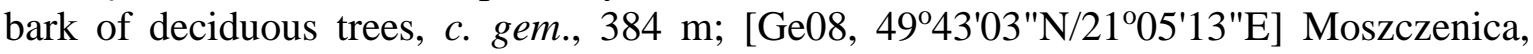
'Księży Las' forest, mineral soil in Abies alba forest, c.gem., 365 m.

Sanionia uncinata (Hedw.) Loeske - [Ge08, 49 43'03"N/21 $\left.05^{\circ} 13^{\prime \prime E}\right]$ Moszczenica, 'Księży Las' forest, rotten wood in Abies alba forest, $365 \mathrm{~m}$.

Schistidium crassipilum H.H.Blom - [Fe87, 49 $\left.51^{\prime} 26^{\prime \prime N} / 21^{\circ} 03^{\prime} 01^{\prime \prime E}\right]$ Lubaszowa, near road to

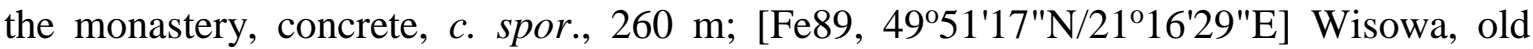
concrete, c. spor., $384 \mathrm{~m}$.

Sciuro-hypnum oedipodium (Mitt.) Ignatov \& Huttunen - [Fe98, 49 49'24"N/21 $\left.{ }^{\circ} 10^{\prime} 28^{\prime \prime E}\right]$ Żurowa, 'Borówka' sandstone outcrop, litter in young Pinus sylvestris forest, $344 \mathrm{~m}$; [Fe88, 49 $\left.50^{\prime} 05^{\prime \prime N} / 21^{\circ} 05^{\prime} 33^{\prime \prime E}\right]$ Jodłówka Tuchowska, between Ratówki and Kobyli Dział settlements, on ground in Abies alba forest, $383 \mathrm{~m}$.

Sciuro-hypnum plumosum (Hedw.) Ignatov \& Huttunen - [Fe87, 49 51'26"N/21 $\left.03^{\circ} 01^{\prime \prime E}\right]$ Lubaszowa, near road to the monastery, boulders in stream, $255 \mathrm{~m}$.

Sciuro-hypnum populeum (Hedw.) Ignatov \& Huttunen - [Fe87, 49 51'26"N/21 $03^{\circ}$ '01"E] Lubaszowa, near road to the monastery, concrete bridge, $255 \mathrm{~m}$.

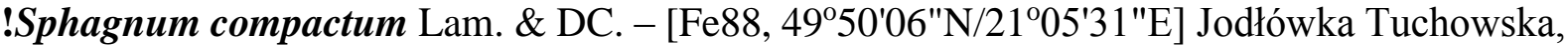
between Ratówki and Kobyli Dział settlements, wet slope in Abies alba forest, $368 \mathrm{~m}$.

:Sphagnum fallax (H.Klinggr.) H.Klinggr. - [Fe97, 4947'53"N/2100'07"E] Przedmieście Wielkie, margin of coniferous forest, $332 \mathrm{~m}$; [Ge08, 49 $\left.43^{\prime} 05^{\prime \prime N} / 21^{\circ} 05^{\prime} 35^{\prime \prime E}\right]$ Moszczenica, 'Księży Las' forest, forest floor in Abies alba forest, $344 \mathrm{~m}$.

!Sphagnum girgensohnii Russow - [Fe97, 4946'41"N/2058'45"E] Ciężkowice, 'Wodospad' nature monument, on ground in Abies alba forest, $300 \mathrm{~m}$; [Fe88, 49 $50^{\prime} 05^{\prime \prime N} / 21^{\circ} 05^{\prime} 33^{\prime \prime} \mathrm{E}$ ] Jodłówka Tuchowska, between Ratówki and Kobyli Dział settlements, on ground in Abies

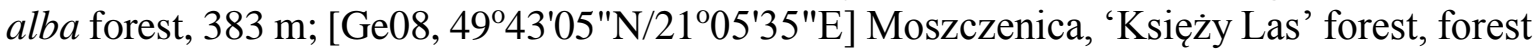
floor in Abies alba forest, $344 \mathrm{~m}$. 
!Sphagnum palustre L. - [Fe97, 49 46'41"N/20'58'45"E] Ciężkowice, 'Wodospad' nature monument, on ground in Abies alba forest, $300 \mathrm{~m}$; [Ge08, 49 $43^{\prime} 05^{\prime \prime N} / 21^{\circ} 05^{\prime} 35^{\prime \prime} \mathrm{E}$ ] Moszczenica, 'Księży Las' forest, forest floor in Abies alba forest, $344 \mathrm{~m}$.

!Sphagnum russowii Warnst. - [Fe97, 4946'41"N/2058'47"E] Ciężkowice, 'Wodospad' nature monument, on ground in Abies alba forest, $297 \mathrm{~m}$.

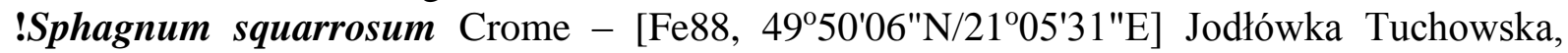
between Ratówki and Kobyli Dział settlements, wet slope in Abies alba forest, c. spor., 368 m; [Ge08, 49 43'05"N/2105'35"E] Moszczenica, 'Księży Las' forest, moist forest floor in Abies alba forest, $344 \mathrm{~m}$.

![R]Syntrichia papillosa (Wilson) Jur. - [Ff90, 49 49'14"N/21 223'25"E] Brzyska, bark of Acer platanoides and Fraxinus excelsior in old park near the castle, c. gem., $241 \mathrm{~m}$.

Syntrichia ruralis (Hedw.) F.Weber \& D.Mohr - [Fe88, 4950'37"N/21 05'50"E] Jodłówka Tuchowska-Ratówki, insolated mineral soil near mountain hut, $501 \mathrm{~m}$; [Fe98, $\left.49^{\circ} 49^{\prime} 36^{\prime \prime N} / 21^{\circ} 11^{\prime} 38^{\prime \prime E}\right]$ Żurowa, Niwy settlement, on the concrete tile on the roof of barn, $280 \mathrm{~m}$.

![R]Syntrichia virescens (De Not.) Ochyra - [Fe98, N 4948'39"; E 2108'29"] Olszyny, bark of wayside Fraxinus excelsior, $290 \mathrm{~m}$.

Tetraphis pellucida Hedw. - Rotten wood in forests, always with gemmae. [Fe89,

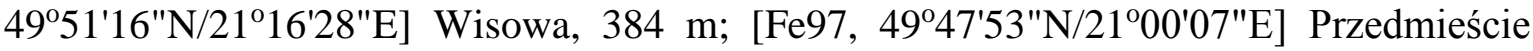
Wielkie, coniferous forest $332 \mathrm{~m}$; [Fe88, 49 $\left.50^{\circ} 05^{\prime \prime N} / 21^{\circ} 05^{\prime} 33^{\prime \prime E}\right]$ Jodłówka Tuchowska, between Ratówki and Kobyli Dział settlements, Abies alba forest, $383 \mathrm{~m}$; [Ge08, 49 $43^{\prime} 04^{\prime \prime N} / 21^{\circ} 05^{\prime 2} 20^{\prime E}$ ] Moszczenica, 'Księży Las' forest, Abies alba forest, c.spor., c.gem., $349 \mathrm{~m}$.

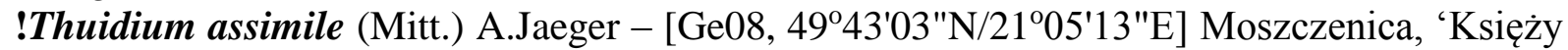
Las' forest, mineral soil at margin of Abies alba forest, $365 \mathrm{~m}$.

!Thuidium tamariscinum (Hedw.) Schimp. - On ground in Abies alba forest. [Fe97, 49 46'41"N/20 58'45"E] Ciężkowice, 'Wodospad' nature monument, $300 \mathrm{~m}$; [Fe88, 49 $50^{\prime} 06^{\prime \prime N} / 21^{\circ} 05^{\prime} 31^{\prime \prime E] ~ J o d ł o ́ w k a ~ T u c h o w s k a, ~ b e t w e e n ~ R a t o ́ w k i ~ a n d ~ K o b y l i ~ D z i a ł ~}$ settlements, $368 \mathrm{~m}$; [Fe90, 4948'13"N/2122'28"E] Brzyska-Strebikowa, 398 m; [Ge08, 49 $43^{\prime} 03^{\prime \prime N} / 21^{\circ} 05^{\prime} 13^{\prime \prime E] ~ M o s z c z e n i c a, ~ ' K s i e ̨ z ̇ y ~ L a s ' ~ f o r e s t, ~} 365 \mathrm{~m}$.

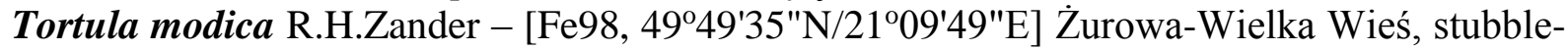
field, c. spor., $305 \mathrm{~m}$.

Tortula muralis Hedw. - [Fe87, 49 51'26"N/21 $\left.03^{\circ} 01^{\prime \prime E}\right]$ Lubaszowa, near road to the

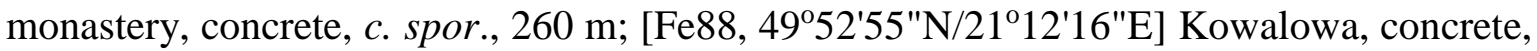
c. spor., $281 \mathrm{~m}$.

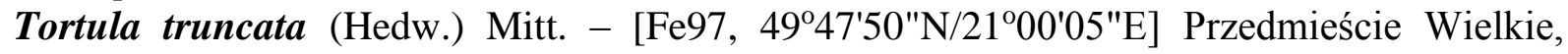
stubble-field, c. spor., $343 \mathrm{~m}$.

[R]Weissia squarrosa (Nees \& Hornsch.) Müll.Hal. - [Ge08, 49 43'03"N/21 $\left.05^{\prime} 13^{\prime \prime E}\right]$ Moszczenica, Księży Las forest, dry ditch at the margin of Abies alba forest, associated with Ceratodon purpureus, Bryum rubens, c.spor., $365 \mathrm{~m}$.

\section{Discussion on most important records}

Among the discovered bryophytes, several are interesting from the chorological point of view in the Polish part of the Carpathians. They are:

Phaeoceros carolinianus - hornwort obviously vanishing from the bryoflora of the Carpathians, mainly due to agriculture abandonment. Recently reported from scattered sites in the lower elevations of the following regions: the Gorce (Mierzeńska 1994), Beskid Wysoki (Klama 1996) and Beskid Mały and Kotlina Żywiecka (Stebel \& Stebel 1998). 


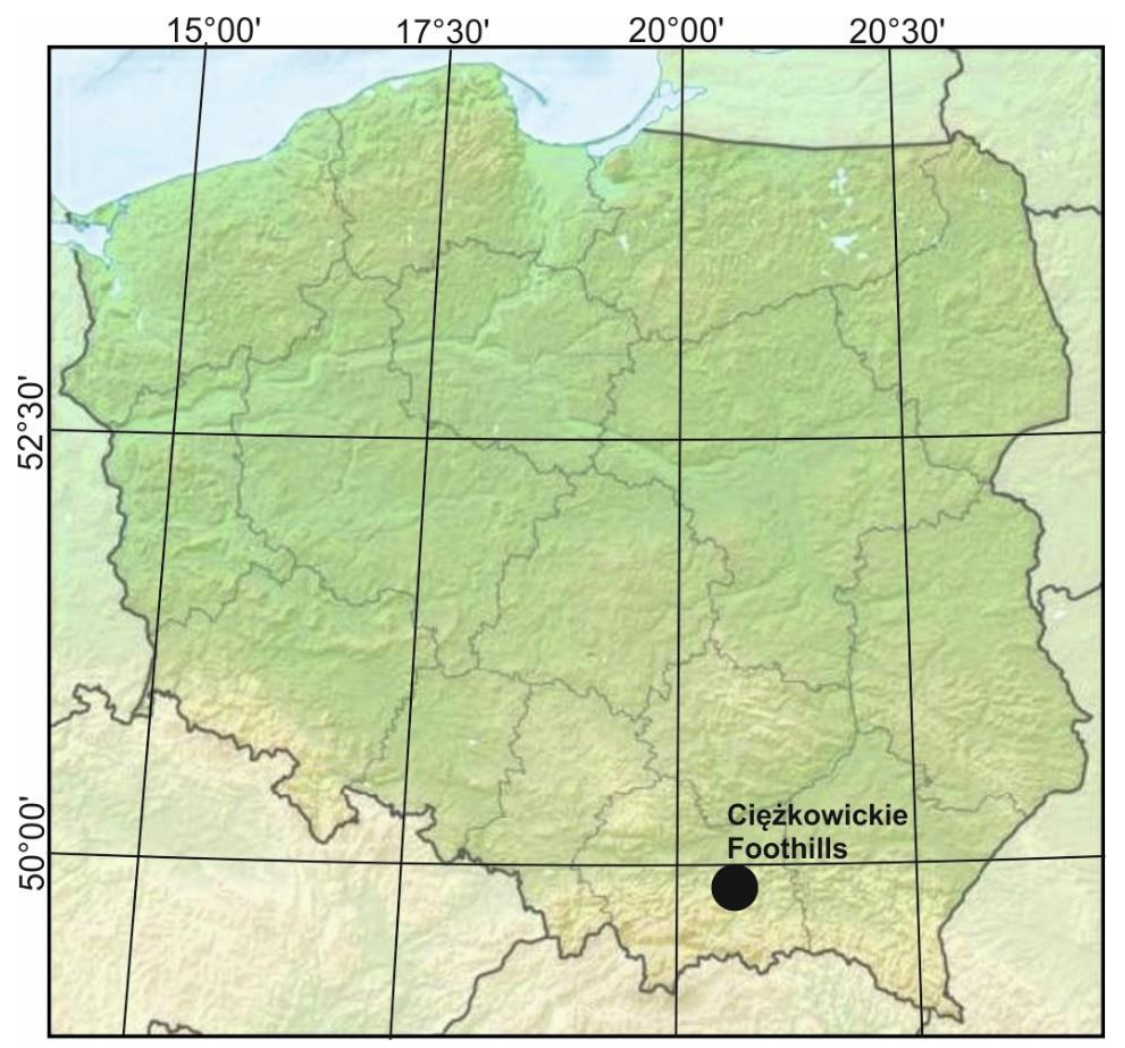

Fig 1: Location of the Ciężkowickie Foothills in Poland.

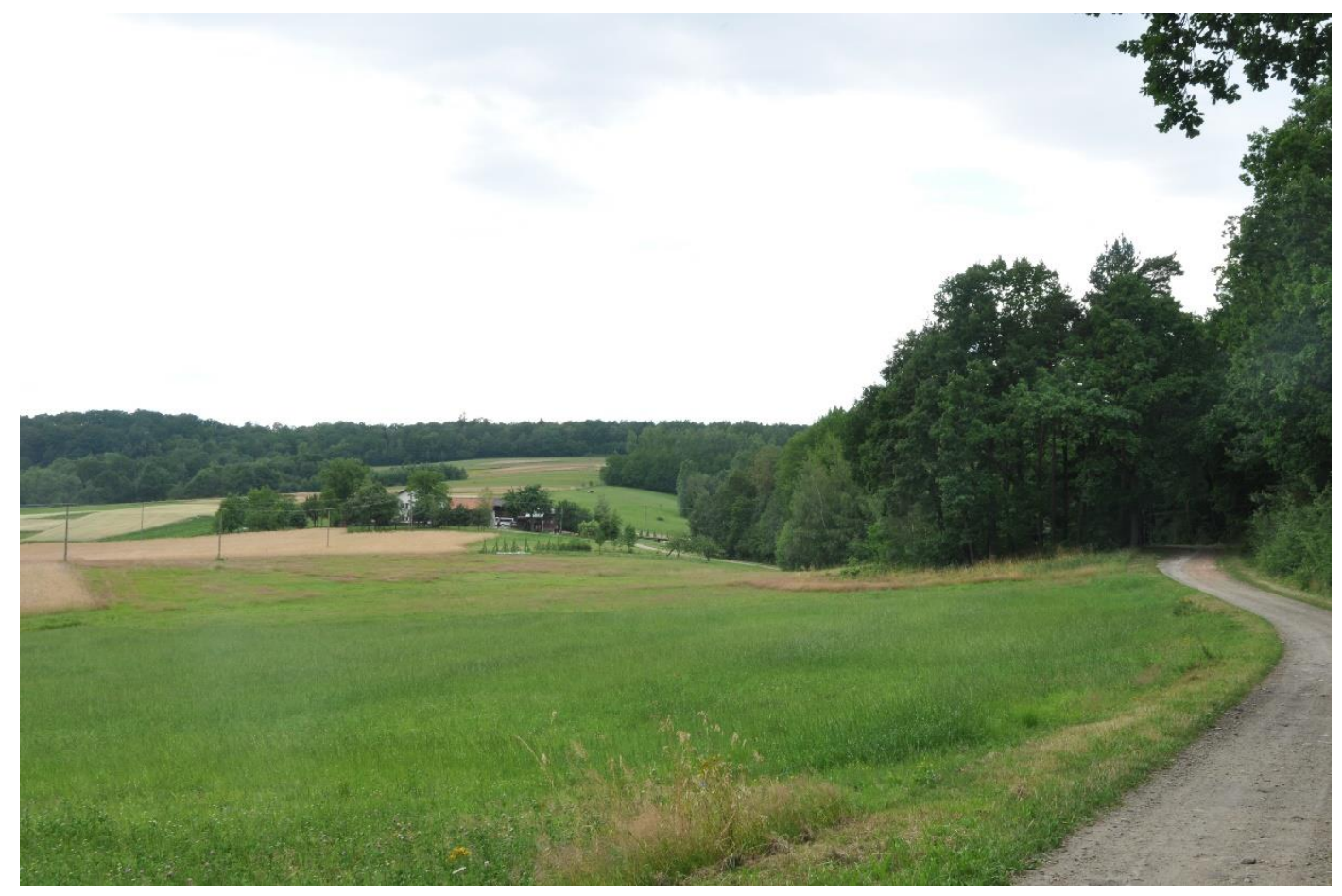

Fig 2: Typical landscape of the Ciężkowickie Foothills. Vicinity of Zadziele settlement near Łużna village (photo by G. Vončina, 9 June 2016). 


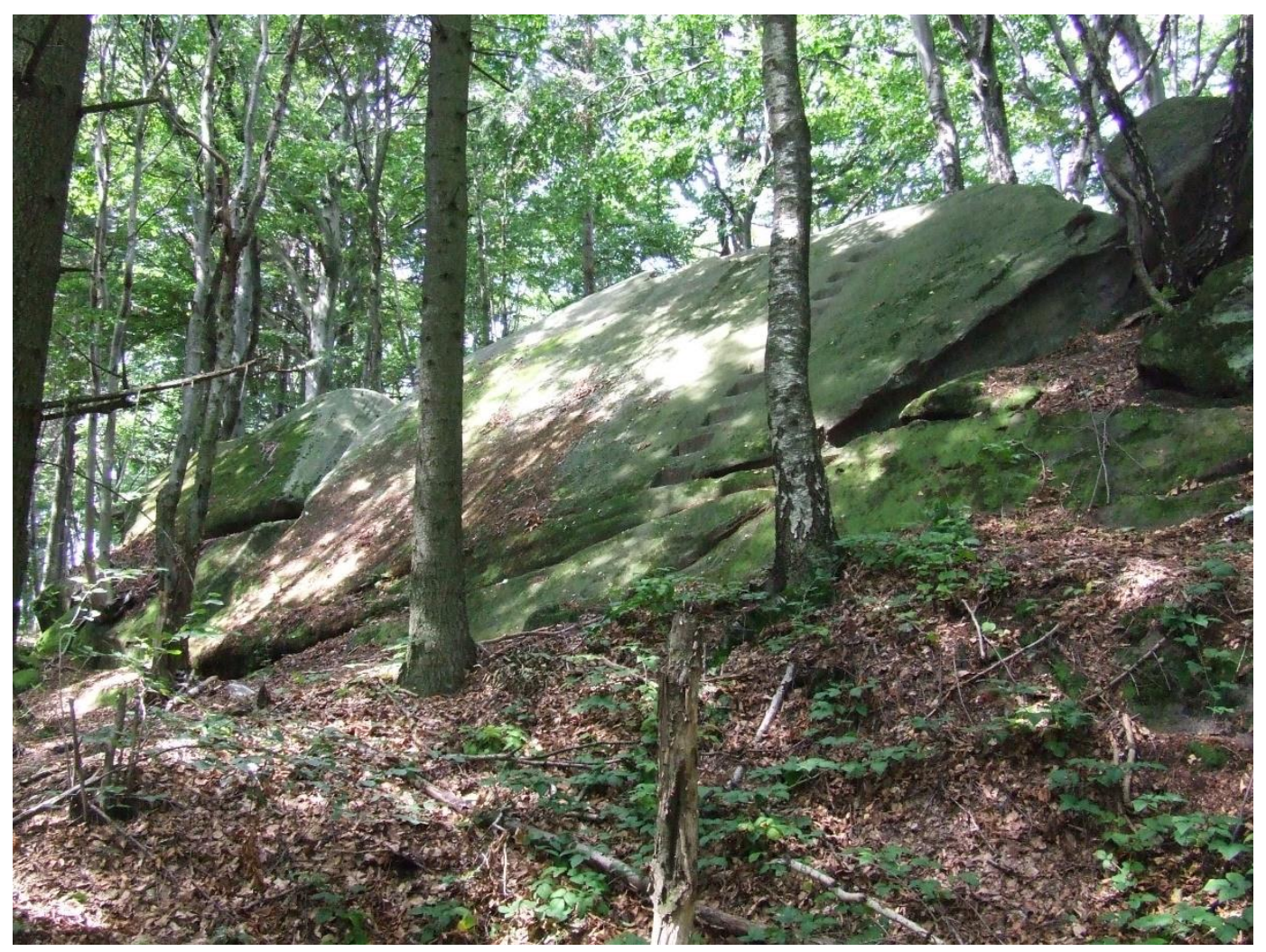

Fig 3: 'Ostry Kamień' sandstone outcrop near Ryglice (photo by A. Stebel, 23 August 2014).

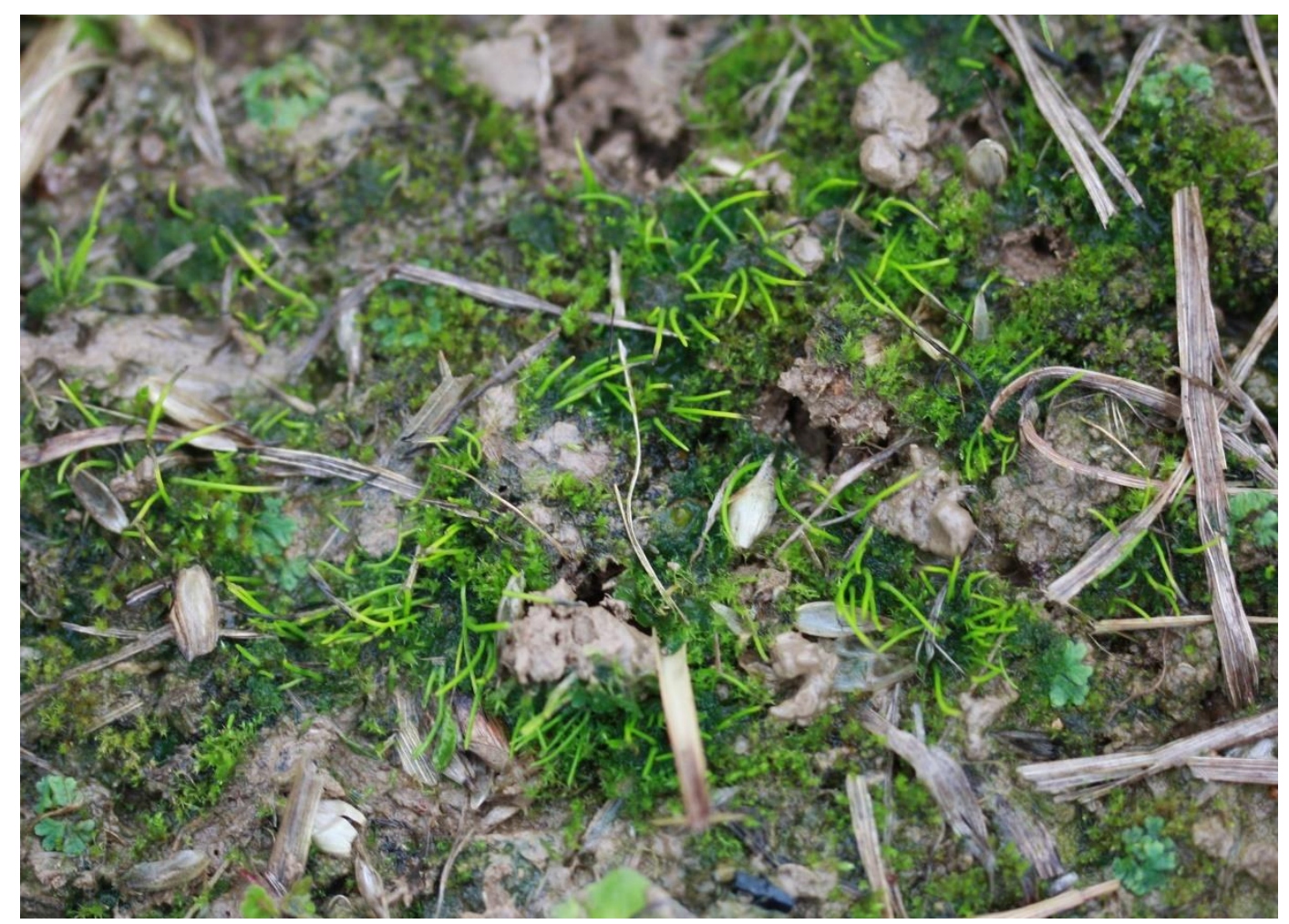

Fig 4: Stubble-field with abundant occurrence of hornworts near Żurowa-Wielka Wieś (photo by A. Stebel, 23 August 2014). 


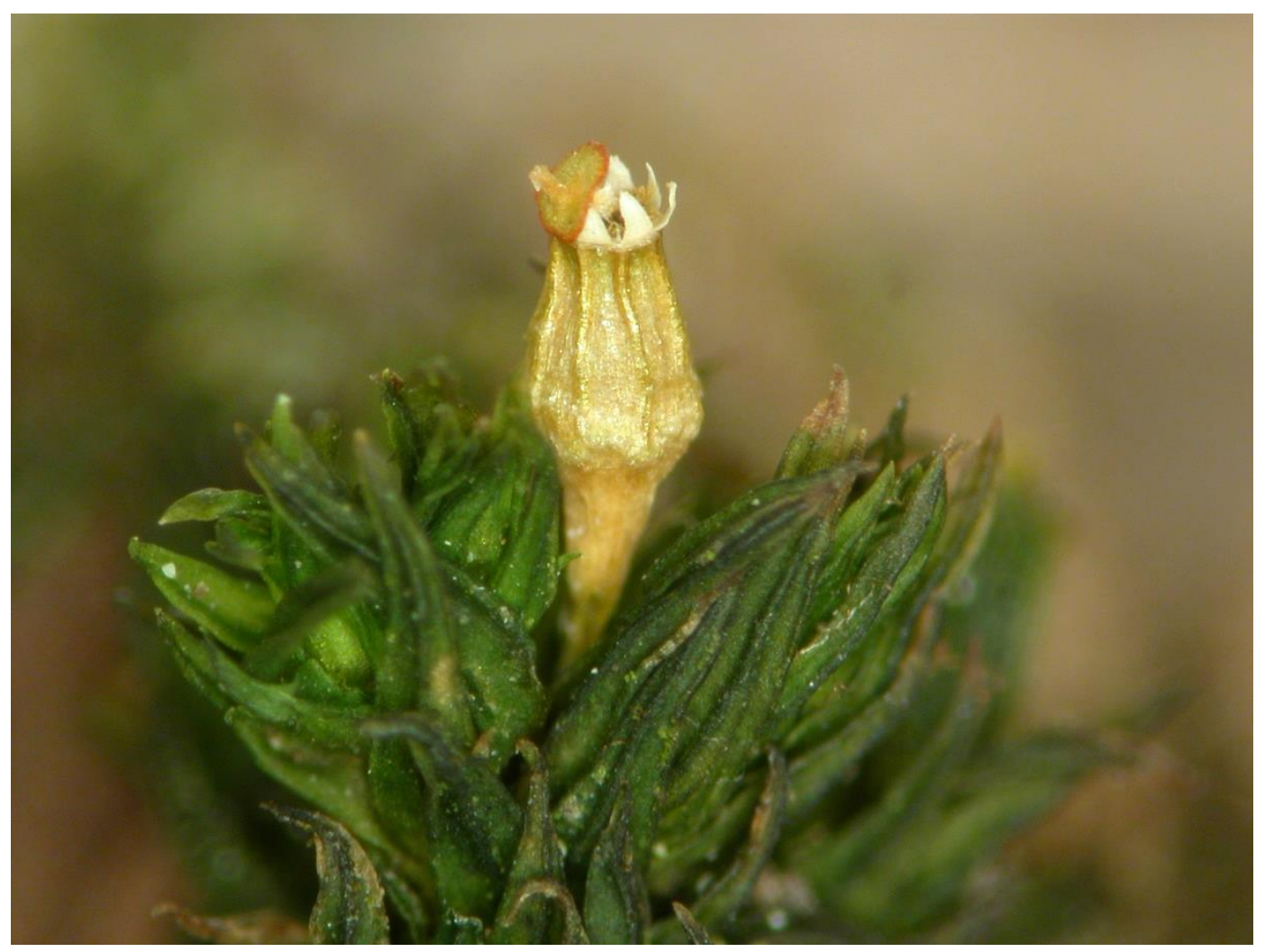

Fig 5: Epiphytic moss Orthotrichum patens (photo by V. Plášek).

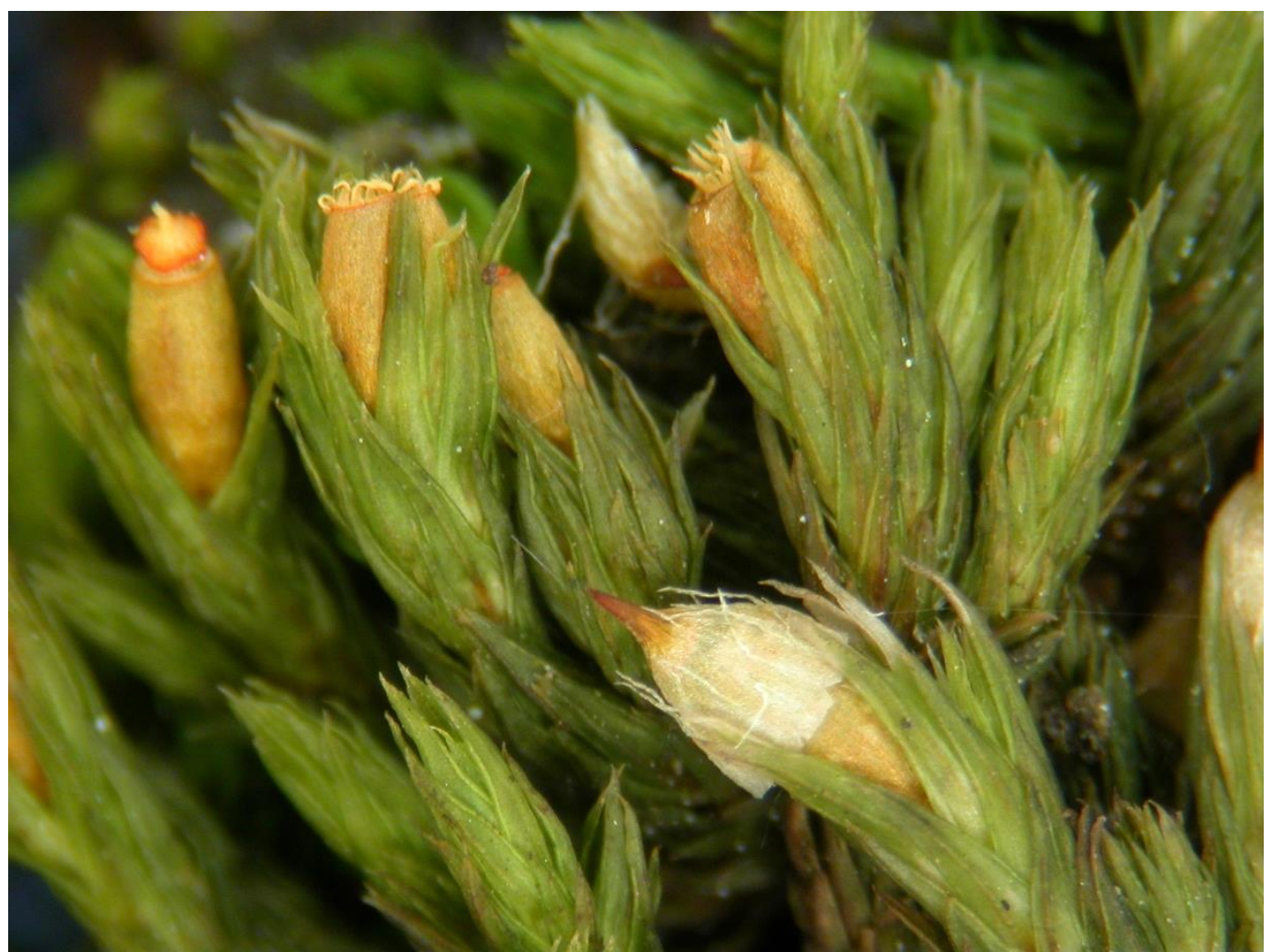

Fig 6: Epiphytic moss Orthotrichum striatum (photo by V. Plášek). 
Campylopus introflexus - invasive moss, known from many localities in the lowland part of Poland, in the Carpathians still rare, but number of its localities quickly increases. Reported from the following regions: the Beskid Mały (Stebel 1995, 2010), Beskid Wysoki (Stebel \& Fojcik 2005), Dynowskie Foothills (Armata 2005), Bieszczady Zachodnie (Stebel \& Żarnowiec 2010), Orawsko-Jordanowskie Foothills (Stebel \& Vončina 2014), Gorce (Stebel et al. 2016) and Rożnowskie Foothills (Stebel 2016a).

Orthotrichum patens (Fig. 5) - rare epiphytic moss, recently found on many sites in various regions of Poland. In the Carpathians known from the following regions: the Beskid Mały (Stebel 2010), Silesian Beskid (Stebel 2006), Pieniny (Stebel et al. 2010), Dynowskie Foothills (Armata 2005), Orawsko-Jordanowskie Foothills (Stebel \& Vončina 2014), Beskid Niski (Stebel 2016b) and Gorce (Stebel et al. 2016).

Weissia squarrosa - very rare species in Poland. For the first time this species was mentioned in the paper by Itzigsohn (1848) from the vicinity of Frankfurt (Oder). After the Second World War Frankfurt was divided between Germany and Poland. Polish eastern part is at present an independent town Słubice thus it is unclear, if the locality was in western or eastern part of Frankfurt. Next locality was published wrongly from the vicinity of the Pyrnik village near Zielona Góra (Limpricht 1874) but the specimens in fact represented Weissia rostellata (Limpricht 1876). Third locality was found in Wrocław (Limpricht 1876), in the place called 'Kratzbusch' (at present a part of the Wrocław Zoological Garden). Until now it has been the only certain locality of Weissia squarrosa in Poland. In the whole Western Carpathians recently Weissia squarrosa was reported from the Czech part of the Silesian Beskid (Plášek \& Stebel 2002).

Acklowedgements. We are grateful to Iwona Bińkowska and Sylwia Wierzcholska for help in location of the place 'Kratzbusch' in Wrocław.

\section{References}

Armata L. (2005): A contribution to the bryoflora of the Pogórze Dynowskie Foothills (Western Carpathians). Annales Universitatis Mariae Curie-Skłodowska, sectio C 60: 101-111.

- (2006): New records of rare and endangered mosses from the Bieszczady Zachodnie Range and the Carpathian Foothills. - Annales Universitatis Mariae Curie-Skłodowska, sectio C, 61(7): 131-139.

Cykowska B. (2006a): Schistostega pennata (Bryopsida, Schistostegaceae) in the Polish Carpathians. - Polish Botanical Journal 51(1): 93-95.

- (2006b). Diplophyllum albicans (Hepaticopsida, Scapaniaceae) in the Polish Carpathians. - Polish Botanical Journal 51(2): 225-227.

Itzigs sohn H. (1848): Ueber die Laubmoosflora der Mark Brandenburg. - Flora 31(14): 225-229.

Klama H. (1996): Wątrobowce (Hepaticae) Beskidu Żywiecko-Orawskiego (Karpaty Zachodnie). - Monographiae Botanicae 79: 1-144.

- (2006a): Systematic catalogue of Polish liverwort and hornwort taxa. In: J. Szweykowski, An annotated checklist of Polish liverworts and hornworts. pp. 83-100. W. Szafer Institute of Botany, Polish Academy of Sciences, Kraków.

- (2006b): Red list of the liverworts and hornworts in Poland. In: Mirek Z., Zarzycki K., Wojewoda W., Szeląg Z. (eds): Red list of plants and fungi in Poland. pp. 21-33. W. Szafer Institute of Botany, Polish Academy of Sciences, Kraków.

Kondracki J. (1994): Geografia Polski. Mezoregiony fizyczno-geograficzne. Warszawa: Wydawnictwo Naukowe PWN.

Limpricht K.G. (1874): Neue Bürger der schlesischen Laubmossflora. - Hedwigia 13(4): 62-63.

- (1876): Nachträge zu den Laub- und Lebermoosen. In: F. Cohn (ed.): Kryptogamen-Flora von Schlesien 1. pp. 414-444. J. U. Kern's Verlag (Max Müller), Breslau.

Mierzeńska M. (1994): Wątrobowce Gorców. - Fragmenta Floristica et Geobotanica Series Polonica 1: $234-$ 346. 
Ochyra R. \& Szmajda P. (1981): La cartographie bryologique en Pologne. In: J. Szweykowski (ed.), New perspectives in bryotaxonomy and bryogeography. Uniwersytet im. Adama Mickiewicza, Seria Biologia 20: $105-110$

Ochyra R., Żarnowiec J. \& Bednarek-Ochyra H. (2003): Census Catalogue of Polish Mosses. Kraków: Institute of Botany, Polish Academy of Sciences.

Plášek V. \& Stebel A. (2002): Bryophytes of the Čantoryjský hřbet range (Czantoria range) and its foothills (Western Carpathians - Czech Republic, Poland). - Čas. Slez. Muz. Opava (A) 51: 1-87.

Regulation of the Minister of the Environment (Poland) of 9 October 2014 on wild species of plants under protection. Journal of Laws (2014), item 1409.

Stebel A. (1995): Campylopus introflexus (Musci, Dicranaceae) found in the Carpathians. - Fragmenta Floristica et Geobotanica 40(2): 909-911.

- (2006): The mosses of the Beskidy Zachodnie as a paradigm of biological and environmental changes in the flora of the Polish Western Carpathians. Habilitation Thesis No. 17/2006. Medical University of Silesia in Katowice and Sorus, Katowice-Poznań, 347 pp.

- (2010): Mosses of the Beskid Mały Range (Western Carpathians). - Materiały Opracowania Centrum Dziedzictwa Przyrody Górnego Śląska 11: 1-142.

- (2015): Contribution to the bryoflora of the Wiśnickie Foothills (Western Carpathians, Poland). - Acta Musei Silesiae Scientiae Naturales 64: 131-139.

- (2016a): Contribution to the bryoflora of the Rożnowskie Foothills (Western Carpathians, Poland). - Fragmenta Naturae 49: 1-13.

- (2016b): Contribution to the moss flora of the Magura National Park (Western Carpathians, Poland). Fragmenta Naturae 49: 14-26.

Stebel A. \& Fojcik B. (2005): Kolejne stanowiska Campylopus introflexus (Bryopsida, Dicranaceae) w województwie śląskim. - Fragmenta Floristica et Geobotanica Polonica 12(2): 412-414.

Stebel A., Krause R. \& Smieja A. (2016): Nowe stanowiska mchów we florze Gorców (Karpaty Zachodnie). - Fragmenta Floristica et Geobotanica Polonica 23(1): 175-177.

Stebel A., Ochyra R. \& Vončina G. (2010): Mosses of the Pieniny Range (Polish Western Carpathians). Poznań: Sorus.

Stebel A. \& Stebel A.M. (1998): Materiały do brioflory Beskidu Małego i północnej części Kotliny Żywieckiej (Karpaty Zachodnie). - Fragmenta Floristica et Geobotanica Series Polonica 5: 217-236.

Stebel A. \& Vončina G. (2014): Bryophyte diversity in the flora of the Orawsko-Jordanowskie foothills (Polish Western Carpathians). Zakopane: Muzeum Tatrzańskie w Zakopanem.

Stebel A. \& Żarnowiec J. (2010): Materiały do flory mchów Bieszczadów Zachodnich (Karpaty Wschodnie). - Roczniki Bieszczadzkie 18: 134-156.

Szafran B . (1956): Zapiski bryologiczne z Karpat Zachodnich (Beskidy, Tatry, Pieniny). - Fragmenta Floristica et Geobotanica 2(1): 143-167.

Żarnowiec J., Stebel A. \& Ochyra R. (2004): Threatened moss species in the Polish Carpathians in the light of a new Red-list of mosses in Poland. In: Stebel A. \& Ochyra R. (eds): Bryological studies in the Western Carpathians. pp. 9-28. Sorus, Poznań.

Author's addresses: Adam Stebel, Department of Pharmaceutical Botany, School of Pharmacy with Division of Laboratory Medicine in Sosnowiec, Medical University of Silesia in Katowice, Ostrogórska 30, 41-200 Sosnowiec, Poland E-mail: astebel@sum.edu.pl

Grzegorz V ončina, Pieniny National Park, ul. Jagiellońska 107b, 34-450 Krościenko nad Dunajcem, Poland

E-mail: gvonvina@poczta.onet.pl 